\title{
Targeted Delivery of Cytotoxic Drugs: Challenges, Opportunities and New Developments
}

\author{
Samuele Cazzamalli, Alberto Dal Corso, and Dario Neri*
}

\begin{abstract}
Cytotoxic drugs, which are commonly used for the pharmacotherapy of many forms of cancer, often cause substantial toxicity to the patient without being able to induce long-lasting remissions. Ligands specific to accessible tumor-associated targets, capable of selective localization at the neoplastic site, may facilitate the preferential delivery of anti-cancer drugs, boosting activity and helping spare normal organs. In this article, we present a critical analysis of the limitation of conventional anti-cancer drugs and we contrast monoclonal antibodies and small organic ligands, as vehicles for pharmacodelivery applications.
\end{abstract}

Keywords: Antibody-drug conjugates · Drug delivery · Small molecule-drug conjugates

The treatment of patients with various forms of cancer (and especially those with disseminated disease) often involves the administration of cytotoxic drugs, which are used to limit the uncontrolled tumor growth and, ideally, induce a remission. Historically, most anti-cancer drugs would bind to nucleic acids or impact on their production, acting as anti-metabolites. Inhibitors of chromatin function or drugs affecting endocrine function are also used. ${ }^{[1]}$ Over the last two decades, new classes of small molecule anti-cancer drugs have entered clinical practice, including kinase inhibitors. ${ }^{[2]}$

It is generally assumed that anti-cancer drugs act directly on tumor cells, but the interaction with endothelial cells ${ }^{[3]}$ or with certain leukocytes (e.g. regulatory T cells) could also contribute to the therapeutic effect.

While chemotherapy can induce longlasting complete remissions in previously incurable disseminated malignancies (e.g. testicular cancer and certain hematological cancer types), the majority of metastatic forms of cancer cannot be cured by cytotoxic agents. One of the main limitations of conventional cancer chemotherapy relates to the inability of small organic drugs to reach tumor cells. Nuclear medicine studies, performed in patients using radiolabeled preparations of anti-cancer agents, have shown that only a small portion of the drug reaches the neoplastic masses in vivo (Fig. 1). ${ }^{[4]}$ These findings are consistent with the results of many quantitative biodistribution investigations, performed in tumor-bearing rodents, which indicate a limited ability of most conventional anticancer drugs to preferentially localize at the site of disease. ${ }^{[5]}$

In an attempt to improve the pharmacokinetic properties of anti-cancer drugs, antibodies, small ligands and polymers have been proposed as pharmacodelivery vehicles, with the aim to preferentially release cytotoxic payloads at the site of dis- ease, sparing normal organs. In this article, we will solely focus on ligand-based drug delivery strategies (i.e. on antibody-drug conjugates (ADCs) and small moleculedrug conjugates (SMDCs); Fig. 2).

\section{Antibody-Drug Conjugates}

Three main facts have prompted researchers to use antibodies as vehicles for drug delivery: i) the exquisite specificity by which they can recognize target antigens; ii) the fact that monoclonal antibodies can be raised against virtually all proteins of interest; iii) previous experience with radiolabeled antibody products, which aimed at concentrating therapeutic radionuclides at the tumor site. Cytotoxic drugs represent a convenient class of nonradioactive anti-cancer payloads.

The main milestones in the development of therapeutic ADCs have been reviewed elsewhere ${ }^{[6,7]}$ and will not be re-

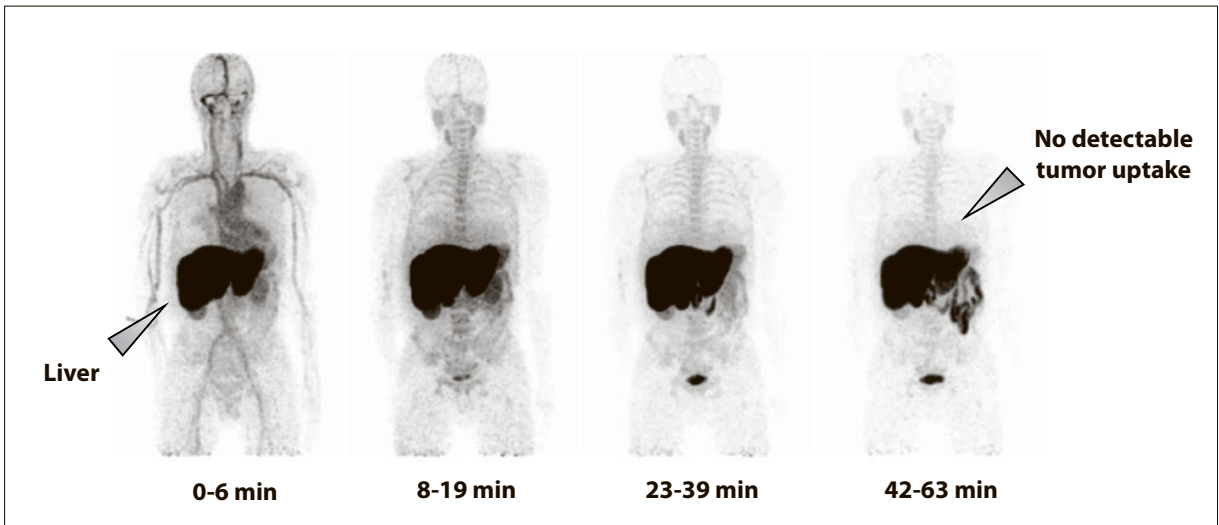

Fig. 1. Positron Emission Tomography imaging of a mesothelioma patient, who received ${ }^{11} \mathrm{C}$-labeled docetaxel. At no time point were the neoplastic lesions visible, while a substantial accumulation of the drug in other organs could be observed. Reproduced from ref. [4] with permission of the Springer International Publishing Group. 


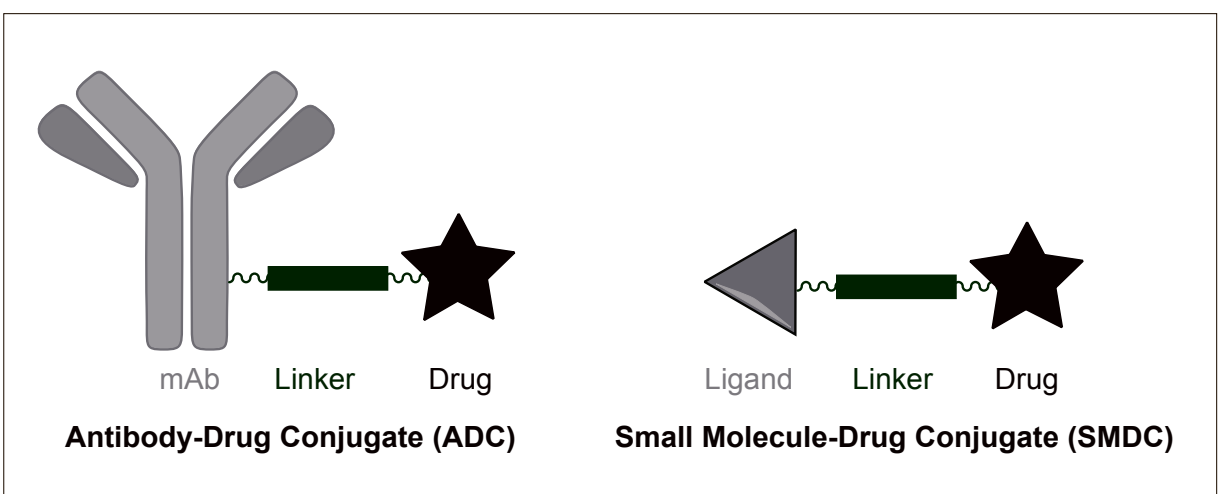

Fig. 2. Schematic representation of the basic elements in antibody-drug conjugates and small molecule-drug conjugates.

peated here. It is important to mention, though, that after the first wave of ADC products, based on low-potency payloads (e.g. doxorubicin, methotrexate derivatives), researchers have rapidly realized that only ultra-potent drugs allow the generation of ADCs, which can be administered at reasonably low dose, for which production costs are still acceptable. An alternative strategy, which is still being pursued by a number of companies, makes use of medium-potency anti-cancer drugs, which are attached in numerous copies to the antibody molecule, by means of multivalent (e.g. dendrimeric) linkers.

In general, ADCs are developed on the basis of tumor-targeting antibodies, directed against an antigen on the surface of the tumor cell, which rapidly internalizes, thus acting as a 'Trojan horse' for the delivery and subsequent release of a cytotoxic payload inside the cell. The strict requirement for ADC internalization has recently been challenged. Evidence of potent activity in preclinical models of cancer has been reported for non-internalizing ADC products, directed against a number of targets, including collagen IV, fibrin, splice isoforms of fibronectin and of tenascin-C. [8]

The therapeutic activity and selectivity of ADC products in preclinical models of cancer can be sensational. For example, brentuximab vedotin (a product marketed under the trade name of Adcetris ${ }^{\mathrm{TM}}$ ) cured tumor-bearing mice at doses as low as $1 \mathrm{mg} /$ $\mathrm{Kg}$, corresponding to $1 / 30^{\text {th }}$ of the maximal tolerated dose. ${ }^{[9]}$ Unfortunately, the therapeutic window of ADC products appears to be less wide in patients, for reasons which are still not completely understood. It is possible that the tumor:organ ratios observed with ADC products in tumor-bearing mice are substantially better, compared to the values observed in patients with cancer. Quantitative biodistribution studies in rodent models and dosimetric findings in Nuclear Medicine trials suggest that this may indeed be the case.

TwoADC products have recently gained marketing authorization: Adcetris ${ }^{\mathrm{TM}}$ (for the treatment of certain forms of Hodgkin's Lymphoma and Anaplastic Large Cell Lymphoma) and Kadcyla ${ }^{\mathrm{TM}}$ (for the second-line treatment of HER2-positive metastatic breast cancer) (Fig. 3). Adcetris ${ }^{\mathrm{TM}}$ was approved on the basis of the high proportion of objective responses, observed in patients, who did not have a therapeutic alternative. ${ }^{[10]}$ These initial findings have later been confirmed in a double-blind Phase III clinical trial, administering the product as consolidation therapy after autologous stem-cell transplantation in patients with Hodgkin's Lymphoma at risk of relapse or progression. [11]

Kadcyla $^{\text {TM }}$ is composed by the antiHER-2 humanized antibody trastuzumab, coupled to the potent maytansinoid DM1 via a non-cleavable linker. It is believed that the payload is released inside the tumor cells, after ADC internalization and subsequent proteolytic digestion of the antibody moiety in an endo-lysosomal compartment.[5] In 2013, Kadcyla ${ }^{\mathrm{TM}}$ re-

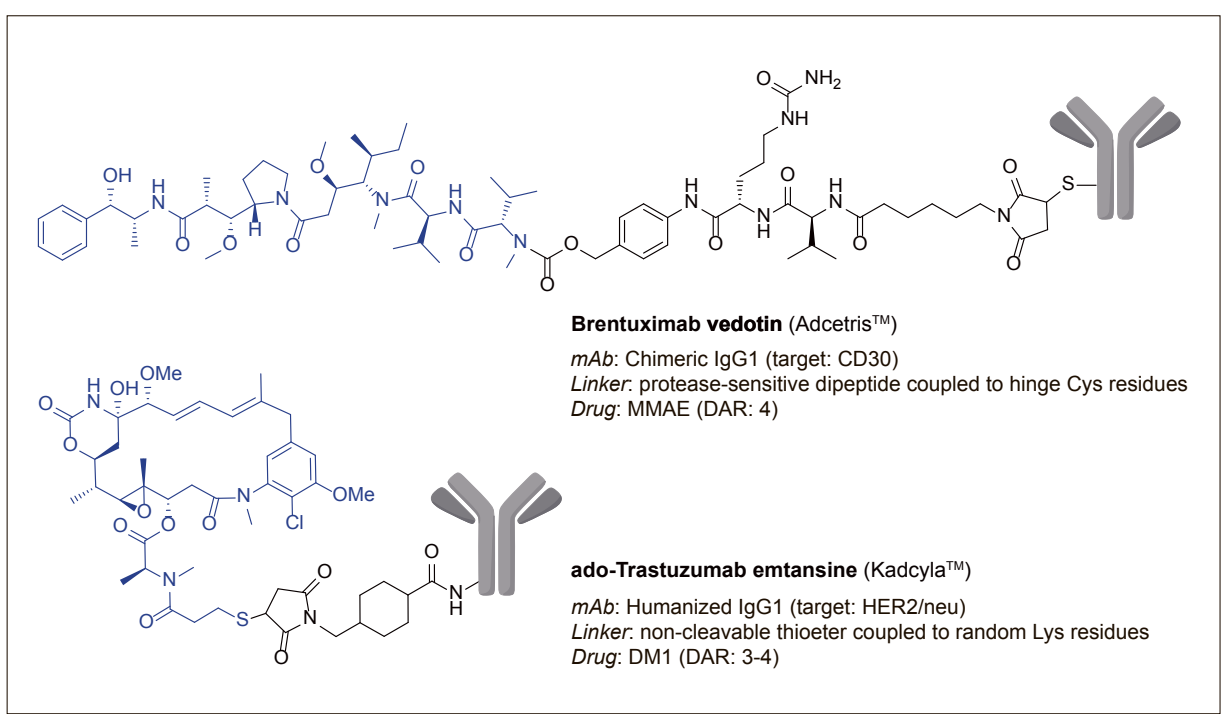

Fig. 3. Schematic representation of the structure of Adcetris ${ }^{\mathrm{TM}}$ and Kadcyla ${ }^{\mathrm{TM}}$. In the first case, monomethyl auristatin E (MMAE), equipped with a cleavable linker, is coupled to the cysteine residues of an anti-CD30 chimeric antibody. In the second case, the potent maytansinoid DM1 is coupled to primary amino groups of the humanized anti-HER2 antibody trastuzumab using a stable linker. ceived FDA approval for the treatment of patients with HER2-positive metastatic breast cancer who had previously received trastuzumab and a taxane, separately or in combination..$^{5]}$ However, the product has recently failed to demonstrate superiority over trastuzumab in combination with standard chemotherapy, in a Phase III clinical trial.

In some mouse models of cancer, antibodies only target perivascular tumor cells, but fail to reach deeper structures within the neoplastic mass, due to the so-called 'antigen barrier' ${ }^{[12]}$ Limited information is available, regarding the homogeneity of tumor-targeting in humans, as these experiments require access to cancer biopsies. Not all ADC products are compatible with a 'bystander effect', whereby the toxic payload could migrate from the initial site of tumor killing and attack neighboring cancer cells.

The majority of ADC products that have been investigated in the clinic exhibited certain levels of bone marrow toxicity, even though the cognate antigen was not expressed in that compartment. It is likely that premature drug release may contribute to these side effects. In addition, complete responses have been reported in patients treated with Adcetris ${ }^{\mathrm{TM}}$, who did not exhibit a detectable expression of the cognate CD30 antigen. ${ }^{[13]}$ It is not clear, at this moment in time, whether the observation of ADC activity in antigen-negative patients is due to technical problems in the execution of immunohistochemical experiments or to an intrinsic property of the antibodydrug conjugate, which circulates in blood for several days and slowly releases its cytotoxic payload. 


\section{Small Molecule-Drug Conjugates}

Antibodies in IgG format exhibit a long circulatory half-life (mediated by interaction with the neonatal Fc receptor FcRn) and extravasate slowly. As a result, acceptably high tumor:organ ratios are observed in vivo only several days after intravenous administration. ${ }^{[14]}$ The suboptimal biodistribution properties of immunoglobulins have prompted researchers to use small organic ligands as alternative vehicles for drug delivery applications. Unlike antibodies, small organic molecules can get out of blood vessels in a matter of seconds. ${ }^{[15]}$ In the absence of a suitable binding event (e.g. recognition of a membrane antigen or of an extracellular matrix component), small molecules rapidly diffuse back into blood and can be excreted. However, small ligands with a sufficiently high binding affinity to accessible tumor-associated antigens can exhibit excellent tumor:blood and tumor:organ ratios, combining a rapid extravasation, a rapid elimination and a long residence time at the site of disease. ${ }^{[5,16]}$ Suitable ligands for drug delivery applications include folate analogues, ${ }^{[16,17]}$ prostate-specific membrane antigen (PSMA) ligands ${ }^{[18]}$ and binders to carbonic anhydrase IX (CAIX). ${ }^{[19]}$

A folate-vinblastin conjugate, developed by Endocyte, exhibited encouraging clinical results for the treatment of advanced ovarian cancer, especially in patients with high uptake of a radiolabeled folate derivative used as companion diagnostic. ${ }^{20]}$ These data led to a conditional approval in Europe, but product development activities were later discontinued on the basis of disappointing Phase III trial results.

Encouraging preclinical results with PSMA ligand derivatives ${ }^{[19]}$ have motivated Endocyte to develop an SMDC product for the treatment of advanced prostate. The drug conjugate EC1169 makes use of a very potent tubulysin payload and is currently being investigated in a Phase I clinical trial, together with a companion diagnostic product. Therapeutic radionuclides have been considered as an alternative avenue for arming PSMA ligands. A recent clinical report on 148 patients with metastatic castration-resistant prostate cancer, treated with the ${ }^{177} \mathrm{Lu}$-labeled-PSMA-617 radiopharmaceutical, evidenced favorable safety and excellent efficacy, with $>40 \%$ of subjects enjoying an objective biochemical and radiological response. ${ }^{[21]}$

Carbonic anhydrase IX is a homodimeric membrane protein, which shows restricted expression in normal tissues, mainly limited to certain structures in the gastro-intestinal tract, but intense expression in renal cell carcinoma and in a number of other malignancies. CAIX does not internalize upon binding with small heteroaromatic sulfonamides and SMDC products, featuring acetazolamide as tumor-homing moiety, exhibit a potent anti-cancer activity in preclinical models. Both DM1 (the payload of Kadcyla ${ }^{\mathrm{TM}}$ ) and MMAE (the payload of Adcetris ${ }^{\mathrm{TM}}$ ) have been coupled to acetazolamide using linkers that can be cleaved in the extracellular space. The corresponding products exhibited a promising anti-cancer activity, ${ }^{[19,22]}$ which was superior to that of sunitinib and sorafenib (two standard drugs for the treatment of metastatic kidney cancer) in tumor-bearing mice. As for SMDCs targeting the folate receptor or PSMA, the tumor targeting performance of CAIX ligands can be monitored using ${ }^{99 \mathrm{~m}}$ Tc-labeled companion diagnostics. ${ }^{[22,23]}$

Other targets for the development of SMDC products include somatostatin receptors, the aminopeptidase CD13 and certain integrins. Unlike antibodies, which can be raised against virtually any antigen, small molecule protein ligands can be difficult to generate, depending on the nature of the cognate target. Nowadays, fully human monoclonal antibodies can be rapidly isolated from large combinatorial phage display libraries, containing billions of different specificities. ${ }^{[24]}$ The advent of DNA-encoded combinatorial chemical libraries allows the creation and screening of compound collections of unprecedented size, ${ }^{[25-30]}$ thus facilitating ligand discovery. These libraries are composed of small organic molecules, individually attached to distinctive DNA fragments serving as amplifiable identification barcodes. Members of DNA-encoded combinatorial libraries can be screened simultaneously by selection with the target protein of interest, immobilized on a solid support. Preferential binders are identified by high-throughput DNA sequencing techniques.

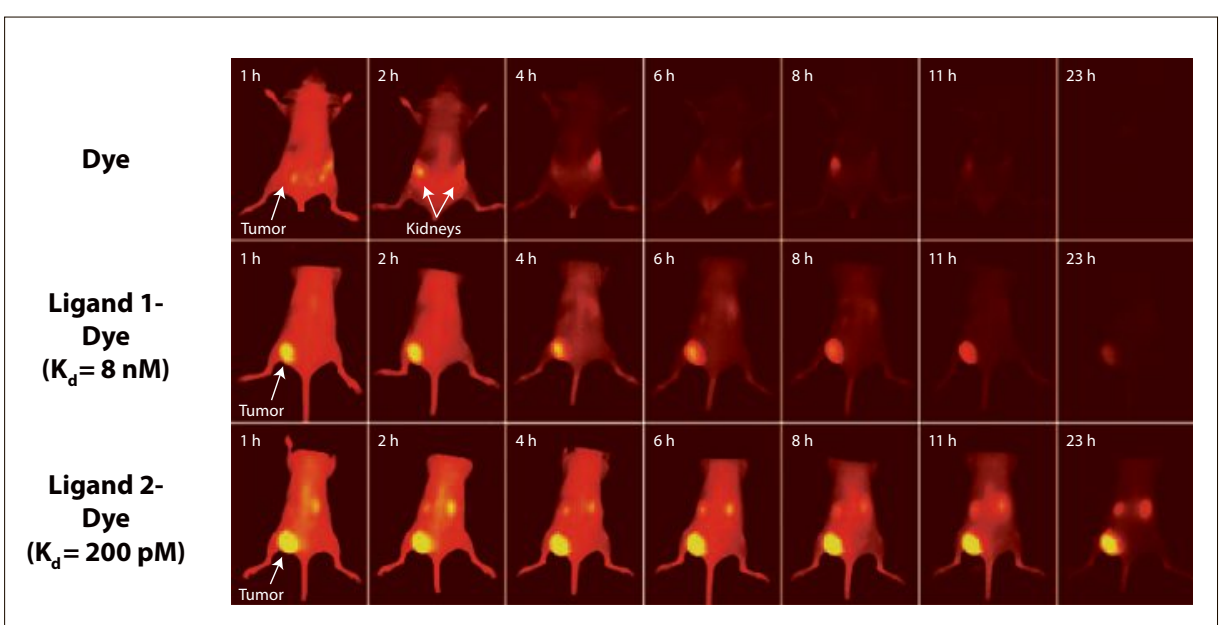

Fig. 4. Near-infrared fluorescence imaging of tumor-bearing mice, imaged at various time points following intravenous injection of a dye, or of the same dye coupled to two different CAIX ligands, differing in binding affinity to the target. Adapted from ref. [31].
The role that DNA-encoded chemical library technology can play for the development of SMDC products is illustrated in the following example. Mice bearing subcutaneously-grafted renal cell carcinoma xenografts were injected with a nearinfrared fluorescent dye or with dye derivatives, coupled to acetazolamide or to a high affinity acetazolamide derivative (Kd $=200 \mathrm{pM}$ ), isolated from a DNA-encoded chemical library[31] (Fig. 4). Fluorescence imaging procedures revealed that the unconjugated dye was not able to localize to the tumor and was rapidly excreted, in full analogy to the behavior of standard chemotherapeutic drugs, illustrated in Fig. 1. By contrast, the dye conjugate with acetazolamide revealed a rapid and selective tumor targeting, which was visible already 1 hour after intravenous administration. The intermediate affinity of the conjugate towards CAIX $(\mathrm{Kd}=8 \mathrm{nM})$ did not allow a long residence time on the neoplastic lesion. By contrast, the higher affinity CAIX targeting agent, exhibited a rapid, longlasting and selective accumulation in the tumor mass (Fig. 4). Similar results were obtained in quantitative biodistribution studies, using the same ligands equipped with a technetium-99m chelator and radioactive counting techniques. ${ }^{[23]}$

\section{Future Perspectives}

ADCs and SMDCs are prodrugs with the potential to substantially improve the therapeutic index of the corresponding cognate drug. Numerous products (especially ADCs) are currently being investigated in clinical trials, even though many companies are reducing their research and development activities in the field, after substantial initial investments. One of the main unresolved challenges, which com- 
plicates the clinical and industrial development of ligand-based targeted cytotoxics, relates to the difficulty in translating promising preclinical results (i.e. cancer cures in tumor-bearing mice) into a corresponding clinical activity. A reliable quantification of the concentration of cytotoxic agent delivered to neoplastic lesions in mouse and man will be of fundamental importance, in order to rationally advance ADC and SMDC research.

Nuclear medicine imaging studies will be extremely useful, in order to quantify product uptake. In particular, it will be important to learn whether lesions that benefit most from pharmacological treatment are also the ones with the highest product uptake. At present, these studies are hindered both by the complexity of coordinating oncology and nuclear medicine investigations in the same patient and by regulatory aspects (e.g. GMP preparations both for the unlabeled and labeled products).

Until now, clinical-stage ADC and SMDC products have rarely demonstrated an ability to induce cancer cures in patients, when used as monotherapy. The opportunity to combine targeted cytotoxics with certain immunostimulatory drugs is becoming increasingly attractive, also in view of the growing role played by immune-oncology drugs for the treatment of patients with metastatic disease. The antibody-based delivery of interleukin-2 (IL2, a potent activator of T cells and NK cells) has been shown to potentiate chemotherapy in preclinical models of cancer, especially when administered after the cytotoxic agent. ${ }^{[32]}$ Similarly, the anti-cancer activity of $\mathrm{ADCs}^{[33,34]}$ and $\mathrm{SMDCs}^{[35]}$ has been shown to be potentiated by tumortargeting antibody-IL2 fusion proteins. It is likely that the tumor becomes more immunogenic, after the insult caused by the action of cytotoxics. Possible mechanisms may involve the up-regulation of stress surface proteins on tumor cells (e.g. MIC-A) and the subsequent recognition by NK cells, or the increased presentation of tumor-associated peptides on MHC class I molecules.

Looking into the future, we anticipate that ADCs and SMDCs may play an increasingly important role not only in oncology, but also for the delivery of other classes of drugs (e.g. antibiotics, anti-inflammatory drugs). ${ }^{[36]}$ In our opinion, the therapeutic potential of ligands coupled to small organic drugs will be best exploited in combination with innovative immunomodulatory modalities, such as immunological check-point inhibitors or antibody- cytokine fusion proteins. ${ }^{[37,38]}$ Finding the right combination partners will require substantial research efforts, but may pave the way to therapeutic modalities with unprecedented potency and selectivity.

\section{Acknowledgements}

We thank the Swiss National Science Foundation and the European Research Council for financial support.

Received: June 7, 2017

[1] W. B. Pratt, R. W. Ruddon, W. D. Ensminger, J. Maybaum, 'The Anticancer Drugs', Oxford University Press, New York, 1994.

[2] D. Fabbro, Mol. Pharmacol. 2015, 87, 766.

[3] R. S. Kerbel, Science 2006, 312, 1171

[4] A. A. M. Van der Veldt, N. H. Hendrikse, E. F. Smit, M. P. J. Mooijer, A. Y. Rijnders, W. R. Gerritsen, J. J. M. Van der Hoeven, A. D. Windhorst, A. A. Lammertsma, M. Lubberink, Eur. J. Nucl. Med. Mol. Imaging 2010, 37, 1950.

[5] N. Krall, J. Scheuermann, D. Neri, Angew. Chem. Int. Ed. 2013, 52, 1384.

[6] R. V. Chari, M. L. Miller, W. C. Widdinson, Angew. Chem. Int. Ed. 2014, 53, 3796.

[7] E. L. Sievers, P. D. Senter, Annu. Rev. Med. 2013, 64, 15.

[8] R. Gébleux, M. Stringhini, R. Casanova, A Soltermann, D. Neri, Int. J. Cancer 2017, 140 , 1670.

[9] S. Doronina, B. E. Toki, M. Y. Torgov, B. A Mendelsohn, C. G. Cerveny, D. F. Chace, R. L. DeBlanc, R. P. Gearing, T. D. Bovee, C. B. Siegall, J. A. Francisco, A. F. Wahl, D. L. Meyer, P. D. Senter, Nat. Biotech. 2003, 7, 778.

[10] J. Katz, J. E. Janik, A. Younes, Clin. Cancer Res. 2011, 17, 6428.

[11] C.H. Moskowitz, A. Nademanee, T. Masszi, E. Agura, J. Holowiecki, M. H. Abidi, A. I. Chen, P. Stiff, A. M. Gianni, A. Carella, D. Osmanov, V. Bachanova, J. Sweetenham, A. Sureda, D. Huebner, E. L. Sievers, A. Chi, E. K. Larsen, N. N. Hunder, J. Walewski, AETHERA Study Group, Lancet 2015, 385, 1853 .

[12] M. S. Dennis, H. Jin, D. Dugger, R. Yang, L. McFarland, A. Ogasawara, S. Williams, M. J. Cole, S. Ross, R. Schwall, Cancer Res. 2007, 67, 254.

[13] N. L. Bartlett, M. R. Smith, T. Siddiqi, R. H. Advani, O. A. O'Connor, J. P. Sharman, T. Feldman, K. J. Savage, A. R. Shustov, C. S. Diefenbach, Y. Oki, M. C. Palanca-Wessels, M. Uttarwar, M. Li, J. Yang, E. D. Jacobsen, Leuk. Lymphoma 2017, 58, 1607.

[14] G. A. van Dongen, G. W. Visser, M. N. Lub-de Hooge, E. G. de Vries, L. R. Perk, Oncologist 2007, 12, 1379.

[15] G. M. Thurber, K. S. Yang, T. Reiner, R. H. Kohler, P. Sorger, T. Mitchison, R. Weissleder, Nat. Commun. 2013, 4, 1504

[16] M. Srinivasarao, C. V. Galliford, P. S. Low, Nat. Rev. Drug Discov. 2015, 14, 203.

[17] P. S. Low, W. A. Henne, D. D. Doornewerd, Acc. Chem. Res. 2008, 41, 120.

[18] S. A. Kularatne, C. Venkatesh, H. K. Santhapuram, K. Wang, B. Vaitilingam, W. A. Henne, P. S. Low, J. Med. Chem. 2010, 53, 7767.
[19] N. Krall, F. Pretto, W. Decurtins, G. J. Bernardes, C. Supuran, D. Neri, Angew. Chem. Int. Ed. 2014, 53, 4231

[20] R. T. Morris, R. N. Joyrich, R. W. Naumann, N. P. Shah, A. H. Maurer, H. W. Strauss, J. M. Uszler, J. T. Symanowski, P. R. Ellis, W. A. Harb, Annals Oncol. 2014, 25, 852

[21] K. Rahbar, H. Ahmadzadehfar, C. Kratochwil, U. Haberkorn, M. Schäfers, M. Essler, R. P. Baum, H. R. Kulkarni, M. Schmidt, A. Drzezga, P. Bartenstein, A. Pfestroff, M. Luster, U. Lützen, M. Marx, V. Prasad, W. Brenner, A. Heinzel, F. M. Mottaghy, J. Ruf, P. T. Meyer, M. Heuschkel, M. Eveslage, M. Bögemann, W. P. Fendler, B. J. Krause, J. Nucl. Med. 2017, 58, 85.

[22] S. Cazzamalli, A. Dal Corso, D. Neri, Mol. Cancer Ther 2016, 15, 2926.

[23] N. Krall, F. Pretto, M. Mattarella, C. Müller, D. Neri, J. Nucl. Med. 2016, 57, 943.

[24] G. Winter, A. D. Griffiths, R. E. Hawkins, H Hoogenboom, Аnпu. Rev. Immunol. 1994, 12, 433.

[25] S. Brenner, R. A. Lerner, Proc. Natl. Acad. Sci. U.S.A. 1992, 89, 5381.

[26] Z. J. Gartner, B. N. Tse, R. Grubina, J. B. Doyon, T. M. Snyder, D. R. Liu, Science, 2004, 305, 1601 .

[27] R. Franzini, D. Neri, J. Scheuermann, Acc. Chem. Res. 2015, 47, 1247

[28] M. A. Clark, R. A. Acharya, C. C. AricoMuendel, S. L. Belyanskaya, D. R. Benjamin, N. R. Carlson, P. A. Centrella, C. H. Chiu, S. P. Creaser, J. W. Cuozzo, C. P. Davie, Y. Ding, G. J. Franklin, K. D. Franzen, M. L. Gefter, S. P. Hale, N. J. Hansen, D. I. Israel, J. Jiang, M. J. Kavarana, M. S. Kelley, C. S. Kollmann, F. Li, K. Lind, S. Mataruse, P. F. Medeiros, J. A. Messer, P. Myers, H. O' Keefe, M. C. Oliff, C. E. Rise, A. L. Satz, S. R. Skinner, J. L. Svendsen, L. Tang, K. van Vloten, R. W. Wagner, G. Yao, B. Zhao, B. A. Morgan, Nat. Chem. Biol. 2009, $5,467$.

[29] R. A. Goodnow Jr., C. E. Dumelin, A. D. Keefe, Nat. Rev. Drug Discov. 2017, 16, 131 .

[30] N. Winssinger, Chimia 2013, 67, 340 .

[31] M. Wichert, N. Krall, W. Decurtins, R. M. Franzini, F. Pretto, P. Schneider, D. Neri, J. Scheuermann, Nat. Chem. 2015, 7, 241

[32] M. Moschetta, F. Pretto, A. Berndt, K. Galler, P. Richter, A. Bassi, P. Oliva, E. Micotti, G. Valbusa, K. Schwager, M. Kaspar, E. Trachsel, H. Kosmehl, M. R. Bani, D. Neri, R. Giavazzi, Cancer Res. 2012, 72, 1814

[33] K. L. Gutbrodt, G. Casi, D. Neri, Mol. Cancer Ther. 2014, 13, 1772

[34] T. List, G. Casi, D. Neri, Mol. Cancer Ther. 2014, 13, 2641.

[35] S.Cazzamalli, D. Neri, unpublished results.

[36] S. M. Lehar, T. Pillow, M. Xu, L. Staben, K. K. Kajihara, R. Vandlen, L. DePalatis, H. Raab, W. L. Hazenbos, J. H. Morisaki, J. Kim, S. Park, M. Darwish, B. C. Lee, H. Hernandez, K. M. Loyet, P. Lupardus, R. Fong, D. Yan, C. Chalouni, E. Luis, Y. Khalfin, E. Plise, J. Cheong, J. P. Lyssikatos, M. Strandh, K. Koefoed, P. S. Andersen, J. A. Flygare, M. Wah Tan, E. J. Brown, S. Mariathasan, Nature 2015, $527,323$.

[37] N. Pasche, D. Neri, Drug Discov. Today 2012 17, 583.

[38] F. Bootz, D. Neri, Drug Discov. Today 2016, 21 , 180. 